\title{
CIUDADANOS INFORMADOS Y EMPODERADOS: CLAVES PARA EL PLENO EJERCICIO DE LOS DERECHOS EN SALUD
}

\author{
Walter Gil-Quevedo ${ }^{1,2, a}$, Elvia Agurto-Távara ${ }^{1,2, b}$, Elizabeth Espinoza-Portilla ${ }^{3, c}$
}

\begin{abstract}
RESUMEN
Actualmente, existe un marco normativo peruano que promueve la difusión de información sobre los derechos de salud de la población. Se han creado instituciones, como la Superintendencia Nacional de Salud, que están encargadas de promover los derechos de salud y supervisar las entidades prestadoras de salud. Pese a muchos logros, como la ampliación de la cobertura del Seguro Integral de Salud, se requiere implementar estrategias para que la población acceda a la información y asesoría de forma oportuna, rápida y completa. Algunas experiencias han mostrado potencial de expansión y crecimiento, como la participación de los ciudadanos en las Juntas de Usuarios de los Servicios de Salud y el Programa de Acciones Integradas para la Promoción de los Derechos en Salud, así como la formación de Intendencias descentralizadas de la Superintendencia Nacional de Salud. Los centros de conciliación y arbitraje, el entrenamiento de agentes comunitarios de salud, la vigilancia ciudadana, e incluso estrategias innovadoras utilizando las tecnologías de información y comunicación como las aplicaciones gratuitas para teléfonos inteligentes, constituyen mecanismos importantes para que los ciudadanos dispongan de mayor información y conocimiento en salud, y así, estar más empoderados, al tener la capacidad de ejercer mayor control sobre las decisiones tomadas sobre su salud.
\end{abstract}

Palabras clave: Derechos del Paciente, Derechos Humanos, Capacitación, Perú (fuente DeCS BIREME).

\section{INFORMED AND EMPOWERED CITIZENS: KEYS TO THE FULL EXERCISE OF HEALTH RIGHTS}

\begin{abstract}
Currently, there is a Peruvian legal framework that promotes the dissemination of information on the health rights of the population. Institutions have been created, such as the National Superintendence of Health, which are in charge of promoting health rights and supervising health-care providers. Despite many achievements, such as extending comprehensive health insurance coverage, it is necessary to implement strategies for the population to access information and advice in a timely, quick and complete manner. Some experiences have shown potential for expansion and growth, such as citizens' participation in Health Service User Councils and the Program of Integrated Actions for the Promotion of Health Rights, as well as the formation of decentralized Intendencies of the National Superintendency of Health. The conciliation and arbitration centers, the training of community health agents, the citizen vigilance, and even innovative strategies using information and communication technologies such as free smartphone applications, are important mechanisms for citizens to have more information and knowledge in health, and thus, to be more empowered, having the capacity to exert more control over the decisions taken on their health.
\end{abstract}

Key words: Patient Rights, Human Rights, Training, Peru (source: MeSH NLM).

\section{INTRODUCCIÓN}

La Constitución Política del Perú, en su artículo $7 .^{\circ}$ indica que: Todos tienen derecho a la protección de su salud, la del medio familiar y la de la comunidad así como el deber de contribuir a su promoción y defensa ${ }^{(1)}$. Luego de casi 25 años después de que se redactara este texto, se han dado amplios pasos en la promoción del derecho en salud. Actualmente existe la Ley que establece los Derechos de los Usuarios de los Servicios de Salud, así como su respectivo Reglamento (2,3). Además, contamos con la Superintendencia Nacional de Salud (SUSALUD), entidad adscrita al Ministerio de Salud, que desde el año 2013 protege los derechos en salud de cada ciudadano y cuenta con recursos especializados para gestionar las quejas y reclamos de los usuarios

\footnotetext{
Facultad de Ciencias Sociales, Universidad Nacional José Faustino Sánchez Carrión. Huacho, Perú.

Escuela de Posgrado, Universidad Nacional José Faustino Sánchez Carrión. Huacho, Perú.

Instituto de Gobierno y de Gestión Pública. Universidad de San Martín de Porres. Lima, Perú.

Licenciado en Sociología, doctor en Sociología; ${ }^{b}$ licenciada en Trabajo Social, doctora en Educación; ' médico; magíster en Demografía y Población. Recibido: 16/03/2017 Aprobado: 19/04/2017 En línea: 28/06/2017
} 
que acuden a los establecimientos de salud; supervisa los establecimientos de salud tanto públicos y privados del Perú y es la unidad entidad en salud con potestad sancionadora a través del proceso administrativo sancionador ${ }^{(2)}$.

El Perú se encuentra, además, en proceso de alcanzar la cobertura universal de salud en el contexto de un sistema de salud basado en los derechos que protejan la salud tanto individual como colectiva. Dentro de los objetivos se encuentra el evitar la catástrofe que implica el riesgo de caer en la pobreza como consecuencia de tratar una enfermedad o recuperar la salud de una persona. Hoy en día, el $83,2 \%$ de la población tiene un seguro de salud ${ }^{(4)}$, y 16 millones cuatrocientos mil peruanos vulnerables y de escasos recursos están afiliados al Seguro Integral de Salud, que incluye un paquete de beneficios con atenciones de salud de alto costo y complejidad.

Con un marco legal establecido, y estando en proceso de superar las brechas de atención en salud, se debe poner a disposición de la ciudadanía, la información que requiere para poder ejercer de manera plena los derechos en salud. Este artículo repasa las recientes iniciativas que se están fomentando para la difusión de información en derechos de salud, así como aquellas que promueven el involucramiento directo de la población, tomando en cuenta los retos y oportunidades que surgen en el proceso progresivo de empoderar a los ciudadanos.

\section{RETOS PARA LA DIFUSIÓN DE INFORMACIÓN SOBRE DERECHOS EN SALUD}

Se debe considerar que el material de difusión sobre derechos de salud debe ser accesible a todos. Se debe elaborar material educativo tanto en español como en los idiomas locales, así como tener herramientas diferenciadas para personas con discapacidad visual, auditiva, entre otras. Justamente, estos grupos son los acuden de forma habitual y frecuente a los establecimientos de salud. SUSALUD, como entidad encargada, debería contar con una oficina o jefatura descentralizada en cada región, que además de informar a la población sobre los derechos en salud, evalúe periódicamente el estado de las postas, centros de salud, hospitales y que reciba los reclamos y quejas de la población y de los profesionales de la salud. Se espera la creación de oficinas descentralizadas dentro del país, como la ya creada Intendencia Macrorregional Norte, que abarca Lambayeque, Tumbes, Piura, Cajamarca, La Libertad y Amazonas. Si bien se ha logrado desarrollar muchas actividades de promoción de derechos, la Intendencia Macrorregional Norte aún está en proceso de implementación y consolidación ${ }^{(5)}$.
Estas actividades podrían redirigir la atención de los gobiernos regionales a los establecimientos de salud del primer nivel, que sufren de múltiples carencias materiales, déficit de profesionales de salud, falta de gestión eficiente, y son justamente los que han sido más olvidados, tomando en cuenta que la falta de conocimientos sobre salud es la principal brecha entre el entendimiento entre los prestadores de salud y la población ${ }^{(6)}$.

\section{JUNTAS DE USUARIOS DE LOS SERVICIOS DE SALUDY EL PROGRAMA DE ACCIONES INTEGRADAS PARA LA PROMOCIÓN DE LOS DERECHOS EN SALUD}

Si bien SUSALUD (7) es el organismo encargado de promover, proteger y defender los derechos en salud de la población peruana, no es suficiente que los esfuerzos sean realizados unilateralmente desde los organismos gubernamentales. Es imprescindible que los ciudadanos se involucren en los procesos de vigilancia de los servicios de salud. Esto está enmarcado en la actual legislación, que promueve el monitoreo ciudadano de la calidad de los servicios de salud. La Ley General de Salud, la Ley de Aseguramiento Universal en Salud, la Ley de Derechos de Participación y Control Ciudadanos y los Lineamientos de Política para la Vigilancia Ciudadana en Salud (aprobado por R.M. 040-2011/ MINSA, del 14 de enero de 2011), son algunos ejemplos del amplio marco normativo que impulsa este tipo de iniciativa. Los lineamientos se resumen en la Tabla $1^{(8,9)}$.

Ante el escaso conocimiento sobre los derechos en salud, se han desarrollado varias estrategias para superar la brecha que implica la falta de información disponible para los usuarios. Una de ellas es el desarrollo de Juntas de Usuarios de los Servicios de Salud (JUS). Las JUS buscan articular tres sectores: SUSALUD, las instituciones administradoras de fondos de aseguramiento en salud (IAFAS), las instituciones prestadoras de servicios de salud (IPRESS) y a los usuarios, a través de la participación ciudadana, considerando los factores culturales y generacionales. Ellas han sido efectivas para empoderar a la población en otros países de Latinoamérica como Costa Rica, mejorando el acceso a los servicios de salud ${ }^{(10)}$. Las JUS no pretenden reemplazar a SUSALUD, sino incrementar el grado de confianza entre la ciudadanía y el Estado, acercando los recursos de información a la población. La implementación de las JUS ha sido productiva, hasta el año 2015 participaron siete juntas, realizando 22 talleres, $y$ capacitando a 200 miembros de JUS en Lima y provincias. Se han realizado 14 campañas y se han ofrecido 8644 orientaciones. Estos datos muestran un panorama 
Tabla 1. Lineamientos de política para la vigilancia ciudadana en salud

\begin{tabular}{|ll}
\hline Lineamiento 1 & $\begin{array}{l}\text { Promover la institucionalización y legitimidad social de los mecanismos de la vigilancia ciudadana, } \\
\text { favoreciendo la articulación y participación ciudadana en los diferentes niveles de gobierno. }\end{array}$ \\
\hline Lineamiento 2 & $\begin{array}{l}\text { Promover el desarrollo de la vigilancia ciudadana en salud, procesos de transparencia y rendición de } \\
\text { cuentas hacia la ciudadanía. }\end{array}$ \\
\hline Lineamiento 3 & $\begin{array}{l}\text { Promover los mecanismos de vigilancia ciudadana, la incorporación de gestión de la información y } \\
\text { comunicación, que garanticen la disponibilidad, oportunidad y confiabilidad. }\end{array}$ \\
\hline Lineamiento 4 & $\begin{array}{l}\text { Establecer la vigilancia ciudadana en salud como un proceso que contribuye a la mejora continua del } \\
\text { sistema de gestión sanitaria en los diferentes niveles organizacionales del sector salud en el contexto de } \\
\text { la descentralización. }\end{array}$ \\
\hline Lineamiento 5 & $\begin{array}{l}\text { Promover la sostenibilidad de los mecanismos de la vigilancia ciudadana en salud a través del compromiso } \\
\text { de los niveles de gobierno nacional, regional y local, en el desarrollo de competencias técnicas, y la } \\
\text { provisión de los recursos necesarios para el cumplimiento de sus propósitos. }\end{array}$ \\
\hline
\end{tabular}

Fuente: CARE Perú. Marco Normativo y Aportes Metodológicos para la Implementación de Procesos de Vigilancia Ciudadana de la Calidad de los Servicios de Salud. $2010^{\left({ }^{(8)}\right.}$ y CARE Perú. Lineamientos Metodológicos para el Fortalecimiento de Capacidad para la Vigilancia Ciudadana de la Calidad de los Servicios de Salud ${ }^{(9)}$.

promisorio para futuras acciones de cooperación entre las JUS y SUSALUD ${ }^{(7)}$. Las JUS también han sido implementadas a través de la Intendencia Macrorregional Norte, incluso, se ha creado la Red de Apoyo Regional a la Junta de Usuarios Lambayeque, que incluye a 16 representantes de instituciones y organizaciones de la sociedad civil ${ }^{(5)}$. Así como la Intendencia Macrorregional Norte, esta experiencia podría ser exitosa en otras regiones del Perú.

Otra interesante estrategia es el Programa de Acciones Integradas para la Promoción de los Derechos en Salud. Desde el año 2013 se han realizado actividades de difusión como campañas informativas, reuniones con IPRESS e IAFAS públicas y privadas, y campañas de difusión en medios de comunicación masiva tanto en Lima como en provincias. Según SUSALUD, se dispone de profesionales de diferentes áreas como medicina, comunicación y derecho, que acuden a las 25 regiones del país para coordinar, ejecutar, evaluar y certificar las acciones propuestas ${ }^{(7)}$

\section{AGENTES COMUNITARIOS DE SALUD: ACTORES CLAVE EN LA COMUNIDAD}

La mayoría de datos obtenidos sobre los derechos en salud han sido extraídos de áreas urbanas. Por ejemplo, en la Encuesta Nacional de Satisfacción de Usuarios de Salud (ENSUSALUD 2016) ${ }^{(11)}$ no se ha incluido a los centros de salud de áreas rurales. Se plantea, entonces, otro reto, que es la falta de presencia de personal que vele por los derechos de las personas en comunidades aisladas, remotas, y en especial aquellas donde la población es predominantemente indígena. Existen estrategias que han entrenado exitosamente a agentes comunitarios de salud para ejercer el rol de monitoreo en estas comunidades, donde no existe profesionales de la salud o de otros sectores. Gracias a que estos agentes son miembros de la misma comunidad, se logra cubrir, en algunos casos, las brechas del idioma y distancia. Las comunidades alejadas se ven en desventaja debido al aislamiento geográfico, la distancia hacia los centros de salud y la falta de información disponible, lo que se agudiza por la falta de medios de comunicación como la telefonía celular o internet.

En el Manual del Agente Comunitario de Salud (12), desarrollado por el Ministerio de Salud, se ha considerado el tema de derechos de salud en un capítulo y se refuerza en otras secciones del material, pero los agentes comunitarios de salud usualmente atienden varios problemas de la comunidad y no siempre logran dedicarse exclusivamente a un tema. Imitando esta estrategia, se debe entrenar a líderes de la comunidad en derechos y deberes en salud, para que se den a conocer en la comunidad, orienten a los miembros de ella para el uso de servicios de la salud y los asesoren en caso exista un caso de vulneración de sus derechos ${ }^{(12)}$.

\section{BUSCANDO MECANISMOS ALTERNATIVOS DE SOLUCIÓN DE CONTROVERSIAS}

La consecuencia de la vigilancia participativa, es la identificación de problemas que, usualmente, deben ser resueltos con celeridad. Existen mecanismos alternativos al proceso judicial, que están dentro de la legislación, que permiten solucionar conflictos y han demostrado ser eficientes tanto en nuestro medio y otros países de Latinoamérica ${ }^{(13)}$. Uno de ellos es el Centro de Conciliación y Arbitraje de la Superintendencia Nacional de Salud, que usa la "conciliación, mediación y 
arbitraje" para procurar soluciones. Esta estrategia tiene un gran potencial y desde el año 2016 es gratuita. Se ha observado un incremento de solicitudes entre 2011 y 2015 en la única oficina del Centro de Conciliación y Arbitraje que existe. Pese a la tendencia positiva, cabe mencionar que aún tiene capacidad de expandirse a través las intendencias macrorregionales o de jefaturas regionales que SUSALUD tiene planeado crear en el futuro. Este último aspecto es imprescindible para poder atender la creciente demanda. Se han atendido el triple de solicitudes en los primeros cuatro meses del 2016, comparado con el año $2015^{(5,14)}$.

La gratuidad de este tipo de servicio y la descentralización de instituciones como SUSALUD, se constituirían en un pilar para la resolución de controversias y conflictos en el sector salud, al promover el acceso a la justicia de los ciudadanos de manera equitativa.

\section{CIUDADANOS INFORMADOS EN LA ERA DE LAS TIC Y EL ACCESO ABIERTO}

Las nuevas tecnologías de información y comunicación (TIC) brindan enormes posibilidades para diseminar información en salud y promover los derechos en salud. El "alfabetismo en salud" o health literacy, es el conjunto de conocimientos individuales que actúan como agente mediador en las toma de decisiones clínicas y de salud en general. El "alfabetismo en salud" es un objetivo de salud pública y un desafío en las estrategias contemporáneas de la educación y comunicación en salud en el contexto actual. Los ciudadanos que posean más conocimiento en salud, estarán más empoderados, al tener la capacidad de ejercer mayor control sobre las decisiones tomadas sobre su salud ${ }^{(15)}$.

En esta era de crecimiento tecnológico incesante, aparece el alfabetismo en salud electrónica (eHealth literacy), que se define como la: Habilidad de las personas de usar tecnologías de información y comunicación emergentes para mejorar la salud o a los cuidados de la salud. El uso de recursos electrónicos cumple un rol importante para que la población acceda de forma rápida a la información, promoviendo toma de decisiones informadas. Sin embargo, las actuales tecnologías de la información y comunicación presentan retos, principalmente, en grupos poblacionales de bajo nivel educativo y de mayor edad. Las personas de menos recursos económicos o en localidades aisladas o rurales, pueden tener acceso limitado o, incluso, no tener acceso a internet, y aquellos de mayor edad pueden no estar familiarizados con el uso de los dispositivos electrónicos modernos (16). Estos últimos puntos deben ser considerados al realizar intervenciones en nuestro país, donde aún existen brechas de acceso a los servicios de comunicación. Por otra parte, es fundamental que los mensajes informativos que se envíen a través de las diversas estrategias que involucren las TIC se validen previamente por la población objetivo, de tal manera que se consideren los diversos aspectos socioculturales y sociotécnicos ${ }^{(17)}$.

A nivel local existen iniciativas para promover el uso de las TIC en la población, como mecanismo de empoderamiento. Por ejemplo, SUSALUD, además de la información que promueve a través de su página web y sus redes sociales, ha desarrollado una aplicación de descarga gratuita para celulares denominada "SUSALUD Contigo" que permite, además de conocer los derechos en salud establecidos en la Ley 29414, consultar qué seguros tiene una persona, buscar establecimientos de salud a nivel nacional, y también permite realizar consultas o quejas que son atendidas por personal especializado ${ }^{(3)}$.

A través de los celulares no solo se permite instalar aplicaciones de descarga gratuita, sino también, es muy fácil obtener una gran cantidad de información de acceso abierto y de calidad (18). Por ejemplo, portales web internacionales de información en español como MedlinePlus en español (https://medlineplus.gov/ spanish/) son excelentes fuentes de información en salud para el público en general. A nivel local, los portales web del Ministerio de Salud (http://www.minsa. gob.pe/) y del Instituto Nacional de Salud (http://www. ins.gob.pe/) ofrecen información de utilidad para el público en general y profesionales de salud.

Es importante mencionar que no toda la información en internet es de fuente confiable, por ello, es necesario considerar criterios de calidad al evaluar la información disponible ${ }^{(19)}$, incluyendo las redes sociales. Asimismo, un mecanismo de empoderamiento al ciudadano también puede brindarse a través de brindar información útil a través de los portales personales de salud, los cuales pueden complementarse de manera muy eficiente con las historias clínicas electrónicas ${ }^{(20,21)}$.

\section{CONCLUSIONES}

Los derechos de salud son únicamente ejercidos por aquellos que los conocen. Los profesionales de la salud deben informar a la población en general de manera adecuada, oportuna y en forma veraz, completa, con amabilidad y respeto sobre sus derechos, sobre las condiciones y características de los servicios de salud, sobre los profesionales de salud que lo atienden, entre otros. Además, los establecimientos deben disponer de medios y procedimientos necesarios y suficientes para garantizar la información a las personas usuarias. Igualmente, es función del Gobierno, a través de sus entidades encargadas como SUSALUD, ofrecer herramientas para el acceso eficiente a la información en derechos de salud, considerando los aspectos 
socioculturales. Para ello, es fundamental promover y realizar estudios cualitativos y abordajes sociotécnicos que incluyan diversas técnicas y metodologías como entrevistas a profundidad, grupos focales, observaciones, etc. que permitan entender mejor las percepciones de los usuarios. En este artículo se han discutido varias estrategias a implementar (incluyendo la necesidad de elaborar material diferenciado, el rol de los agentes comunitarios de salud, las actividades de descentralización), incluso se ha discutido la implementación de innovadoras estrategias utilizando las tecnologías de información y comunicación, a través de las cuales se podría reducir la brecha entre lo ideal y la realidad. Una vez evaluadas urge escalar estas estrategias a nivel nacional.

Contribuciones de los autores: WGQ, EAT y EEP participaron en el diseño, redacción del manuscrito y aprobaron la versión final a publicar. Asimismo, asumen la responsabilidad frente a todos los aspectos del manuscrito.

Fuente de financiamiento: autofinanciado por los autores.

Conflicto de interés: los autores declaran no tener ningún conflicto de interés.

\section{REFERENCIAS BIBLIOGRÁFICAS}

1. Perú, Congreso de la República. Constitución política del Perú. Lima: Congreso de la República; 1993.

2. Perú, Ministerio de Salud. Decreto Supremo 027-2015-SA. Aprueban Reglamento de la Ley que establece los Derechos de las Personas Usuarias de los Servicios de Salud. (13 de agosto de 2015).

3. Perú, Congreso de la República. Ley 29414: Ley que establece los Derechos de las Personas Usuarias de los Servicios de Salud. (30 de setiembre de 2009).

4. SUSALUD. SuReporte. Boletín Informativo. Registro Nominal de Afiliados de Salud al 5 de febrero del 2017. 6 de febrero del 2017.

5. Rebaza-Iparraguirre $\mathrm{H}$, Vela-López M, Villegas-Ortega J, Lozada-Urbano M, Munares-García O. [Challenges to decentralization and local participation within the health rights protection framework: Experience of the SUSALUD Northern Macro-regional Intendance]. Rev Peru Med Exp Salud Publica. 2016 Sep;33(3):556-60.

6. Helitzer D, Hollis C, Cotner J, Oestreicher N. Health literacy demands of written health information materials: an assessment of cervical cancer prevention materials. Cancer Control. 2009 Jan;16(1):70-8.

7. Mezones-Holguín E, Díaz-Romero $\mathrm{R}$, Castillo-Jayme J, Jerí-de-Pinho $M$, Benites-Zapata V, Marquez-Bobadilla E, López-Dávalos C, Philipps-Cuba FM. [Promotion of health rights in Peru: an approach from the perspective of The Peruvian National Health Authority]. Rev Peru Med Exp Salud Publica. 2016;33(3):520-528.

8. CARE Perú. Marco Normativo y Aportes Metodológicos para la Implementación de Procesos de Vigilancia Ciudadana de la Calidad de los Servicios de Salud. 2010.

9. CARE Perú. Lineamientos Metodológicos para el Fortalecimiento de Capacidad para la Vigilancia Ciudadana de la Calidad de los Servicios de Salud.

10. Chávez B, Montoya Y. Comparación de las reformas de los sistemas de salud en Colombia y Costa Rica en su dimensión política. Rev Facult Nac Salud Publica. 2011;29(1):65-74.

11. Perú, INEI. Encuesta Nacional de Satisfacción de Usuarios en Salud [Internet]. Lima: INEI; 2016. [Citado el 15 de marzo de 2017] Disponible en: http://portales.susalud.gob.pe/web/ portal $/ 280$

12. Perú, Ministerio de Salud. Manual del Agente Comunitario de Salud [Internet]. Lima: MINSA; 2009. [Citado el 15 de marzo de 2017] Disponible en: http://bvs.minsa.gob. pe/local/minsa/1024_prom37.pdf

13. Bustamante-Leija LE, MaldonadoCamargo VM, González-Anaya C, Gutiérrez-Vega R. Mecanismos alternativos de solución de controversias en la prestación de servicios de salud. Rev CONAMED. 2016;17(3):126-9.

14. Hidalgo-Salas D, Ortiz-Pérez C, Lobatón-Flores J, Huamaní-Ñahuinlla P, Mezones-Holguín E. [Alternative mechanisms for conflict resolution in the context of health rights: The Peruvian experience from the center for reconciliation and arbitration of the national health authority]. Rev Peru Med Exp Salud Publica. 2016 Sep;33(3):567-73.

15. Nutbeam D. Health literacy as a public health goal: a challenge for contemporary health education and communication strategies into the 21st century. Health Promotion International 2000;15(3):259-267.
16. Neter E, Brainin E. eHealth literacy: extending the digital divide to the realm of health information. J Med Internet Res. 2012;14(1):e19.

17. Kaushik B, Brunette MJ, Fu X, Lui B. Customizable, scalable and reliable community-based mobile health interventions. Proceeding MobileHealth '14. Proceedings of the 4th ACM MobiHoc Workshop on Pervasive Wireless Healthcare. 2014; 43-48. Philadelphia, PA, USA.

18. Lober WB, Flowers JL. Consumer empowerment in health care amid the internet and social media. Semin Oncol Nurs. 2011;27(3):169-82.

19. Curioso WH, Castillo CK, Soto AL, Iglesias QD, Echevarria ZJ, Gotuzzo HE. Cerrando la brecha digital en el Perú: Educando a personas viviendo con VIH/SIDA a evaluar la calidad de la información en salud en la Internet. Rev Peru Med Exp Salud Publica. 2007; 24(3): 280-85.

20. Ball MJ, Smith C, Bakalar RS. Personal health records: empowering consumers. J Healthc Inf Manag 2007;21(1):76-86.

21. Kim EH, Stolyar A, Lober WB, Herbaugh AL, Shinstrom SE, Zierler BK, Soh CB, Kim Y. Challenges to using an electronic personal health record by a low-income elderly population. J Med Internet Res. 2009;11(4):e44.

Correspondencia: Elizabeth Espinoza-Portilla. Dirección: Instituto de Gobierno y de Gestión Pública. Universidad de San Martin de Porres. Calle Martín Dulanto 101, Urb. San Antonio, Miraflores, Lima, Perú.

Teléfono: (+51) 998796750.

Correo Electrónico: espinoza.portilla@gmail.com. 